\title{
Gesund an ungesunden Orten
}

\author{
Psychiatrische Fake-News, richtige und falsche Wahnideen und Patienten, \\ investigativer Journalismus, Psychiatriegeschichte und autobiografische Schnipsel \\ eines Betroffenen
}

\author{
Korrespondenzadresse \\ Prof. Dr. Dr. Manfred Spitzer \\ Universität Ulm \\ Abteilung für Psychiatrie \\ Leimgrubenweg 12-14 \\ $87054 \mathrm{Ulm}$
}

Bibliografie

DOI https://doi.org/10.1055/a-1016-2199

Nervenheilkunde 2020; 39: 125-139

(c) Georg Thieme Verlag KG Stuttgart · New York

ISSN 0722-1541
Im Jahr 1973 wurde im renommierten Fachblatt Science vom an der kalifornischen Universität Stanford arbeitenden Psychologen David Rosenhan (2.11.1929-6.2.2012) eine Studie publiziert, die zu den weltweit meistzitierten Arbeiten aus dem Fachgebiet der Psychiatrie zählt und erstens auf das Fach, zweitens auf die in den Kliniken arbeitenden Menschen und drittens vor allem die von psychischen Krankheiten betroffenen Patienten langfristige gravierende Auswirkungen hatte. Die Arbeit hatte den Titel „On being sane in insane places“ („Wie es ist, gesund an ungesunden Orten zu sein“), und deren Inhalt lässt sich wie folgt kurz zusammenfassen.

\section{Das berühmteste Psychiatrie-Experiment der Welt}

3 gesunde Frauen und 5 gesunde Männer stellten sich unter falschem Nachnamen und unter Angabe falscher sozioökonomischer Daten in 12 psychiatrischen Kliniken vor. Sie berichteten darüber, Stimmen zu hören, die „leer, hohl und dumpf“ sagten. Sie wurden daher unter der Diagnose Schizophrenie (eine Person unter manisch-depressive Störung) in der jeweiligen Klinik aufgenommen, verhielten sich danach jedoch völlig normal. Es dauerte im Mittel 19 Tage (Bereich: 7 bis 52 Tage) bis sie dann wieder - mit der Diagnose Schizophrenie, in Remission - entlassen wurden. Die vielleicht wichtigste Beobachtung der „Pseudopatienten “ bestand darin, dass sie von den Ärzten und dem Pflegepersonal kaum beachtet wurden, wie aus der einzigen Tabelle in der Arbeit hervorgeht ( $\triangleright$ Tab. 1) und beispielhaft in der Studie erläutert wird: Auf die Frage eines Pseudopatienten „Entschuldigen Sie, Dr. X., können Sie mir sagen, wann ich Ausgang habe?“, reagierte der Arzt im Vorbeigehen mit „Guten Morgen, Dave, wie geht es Ihnen heute?“ [25], d. h. er ging weder auf die Frage ein, noch wandte er sich dem Patienten zu.

Die Untersuchung war durchgeführt worden, um die grundlegende Frage zu beantworten, wie man psychische Krankheit von psychischer Gesundheit unterscheiden kann. ${ }^{1}$ Sie fand vor dem Hin-

\footnotetext{
Der erste Satz der Arbeit lautet: „If sanity and insanity exist, how shall we know them" [25].
}

tergrund statt, dass in den 1960er- und 1970er-Jahren des letzten Jahrhunderts die Validität und die Reliabilität psychiatrischer Diagnosen häufig in Zweifel gezogen wurden. In Rosenhans [25] eigenen Worten: „[...] die Verbreitung der Ansicht ist gewachsen, dass die psychologische Kategorisierung von psychischen Erkrankungen im besten Fall nutzlos und im schlimmsten Fall geradezu schädlich, irreführend und abwertend ist."2

Der Text geht wie folgt weiter: „Psychiatrische Diagnosen sind in dieser Sichtweise in den Köpfen der Beobachter und stellen keine validen Zusammenfassungen von Merkmalen der Beobachteten dar. "Unmittelbar anschließend wird dann das Folgende behauptet: „Man kann das Problem angehen, indem man normale Menschen in psychiatrische Kliniken einweisen lässt und dann feststellt, ob und wie sie für gesund befunden wurden. “" 3 Wenig später im Text liest man dann: „Der vorliegende Artikel beschreibt genau dieses Experiment. 8 gesunde Leute erhielten eine geheime Einweisung in $12 \mathrm{Kli}$ niken " 4 in 5 Bundesstaaten an der West- und Ostküste der USA.

Es lohnt sich, die Einzelheiten des Vorgehens etwas genauer zu betrachten [25]: „Nachdem er zur Terminvereinbarung das Krankenhaus angerufen hatte, kam der Pseudopatient in der Klinikaufnahme und schilderte, dass er Stimmen gehört hatte. Auf die Frage, was die Stimmen gesagt hätten, antwortete er, dass sie oft undeutlich seien, er aber Wörter wie ,leer', ,hohl' und ,dumpf' erkannt hätte. [...] Abgesehen von den vorgetäuschten Symptomen und der falschen Angabe von Namen, Beruf und Arbeitsverhältnis wurden keine zusätzlichen Veränderungen der Person, von deren Lebensgeschichte oder deren Lebensumstände vorgenommen. Bedeutsa-

2 \#[...] the view has grown that psychological categorization of mental illness is useless at best and downright harmful, misleading, and pejorative at worst."

3 "Psychiatric diagnoses, in this view, are in the minds of the observers and are not valid summaries of characteristics displayed by the observed. Gains can be made in deciding which of these is more accurate by getting normal people admitted to psychiatric hospitals and then determining whether they were discovered to be sane, and, if so, how."

4 "This article describes such an experiment. Eight sane people gained secret admission to 12 different hospitals." 
> Tab. 1 Deutsche Übersetzung (nach Daten aus [25], Tab 1, Übersetzung durch den Autor). Reaktionen (in Prozent) auf aktive Versuche der Kontaktaufnahme durch die Pseudopatienten mit Psychiatern und Pflegepersonal in psychiatrischen Kliniken im Vergleich zu Kontaktversuchen einer jungen Frau zu Professoren und Ärzten auf dem Campus der Universität Stanford bzw. am dortigen Universitätsklinikum. Die Frau stellte jeweils 6 Fragen (die mittlere Anzahl der beantworteten Fragen ist in der dritten Zeile von unten wiedergegeben).

\begin{tabular}{l} 
Ort \\
Versuch der Kontaktaufnahme mit \\
läuft weiter und dreht sich weg \\
\hline Blickkontakt \\
kurzes Gespräch (pauses and chats) \\
\hline Gespräch (stops and talks) \\
\hline mittlere Anzahl beantworteter Fragen \\
Anzahl der reagierenden Personen \\
Anzahl der Kontaktversuche \\
${ }^{*}$ nicht anwendbar
\end{tabular}

\begin{tabular}{|l|l|}
\hline Psychiatrische Kliniken \\
\hline Psychiater & Pflegepersonal \\
\hline
\end{tabular}

\section{Campus der Universitätsklinik Ärzte Universität}

\section{Professoren}

\begin{tabular}{|l|l|l|}
$\begin{array}{l}\text { "suche einen } \\
\text { Psychiater“ }\end{array}$ & $\begin{array}{l}\text { "suche einen } \\
\text { Internisten“ }\end{array}$ & $\begin{array}{l}\text { keine weitere } \\
\text { Kommentierung }\end{array}$ \\
\hline
\end{tabular}

\begin{tabular}{l|l|}
\hline $71 \%$ \\
\hline $23 \%$ \\
\hline $2 \%$ \\
\hline $4 \%$ \\
\hline
\end{tabular}

$13 \quad 47$

$88 \%$

$10 \%$

$2 \%$

$0,5 \%$

$*$

47

1283

$\%$

\begin{tabular}{|l|l|l|}
\hline $\begin{array}{l}\text { Campus der } \\
\text { Universität }\end{array}$ & Universitätsklinik Ärzte \\
\hline Professoren & $\begin{array}{l}\text { „suche einen } \\
\text { Psychiater“ }\end{array}$ & $\begin{array}{l}\text { „suche ei } \\
\text { Interniste }\end{array}$ \\
\hline $0 \%$ & $0 \%$ & $0 \%$ \\
\hline $0 \%$ & $11 \%$ & $0 \%$ \\
\hline $0 \%$ & $11 \%$ & $0 \%$ \\
\hline $100 \%$ & $78 \%$ & $100 \%$ \\
\hline 6 & 3,8 & 4,8 \\
\hline 14 & 18 & 15 \\
\hline 14 & 18 & 15 \\
\hline
\end{tabular}

\begin{tabular}{|l|l|l|}
$\begin{array}{l}\text { Campus der } \\
\text { Universität }\end{array}$ & \multicolumn{2}{|l}{ Universitätsklinik Ärzte } \\
\hline Professoren & $\begin{array}{l}\text { „suche einen } \\
\text { Psychiater“ }\end{array}$ & $\begin{array}{c}\text { „suche ei } \\
\text { Interniste }\end{array}$ \\
\hline $0 \%$ & $0 \%$ & $0 \%$ \\
\hline $0 \%$ & $11 \%$ & $0 \%$ \\
\hline $0 \%$ & $11 \%$ & $0 \%$ \\
\hline $100 \%$ & $78 \%$ & $100 \%$ \\
\hline 6 & 3,8 & 4,8 \\
\hline 14 & 18 & 15 \\
\hline 14 & 18 & 15 \\
\hline
\end{tabular}

\begin{tabular}{|l|l|l|}
$\begin{array}{l}\text { Campus der } \\
\text { Universität }\end{array}$ & \multicolumn{2}{|l}{ Universitätsklinik Ärzte } \\
\hline Professoren & $\begin{array}{l}\text { „suche einen } \\
\text { Psychiater“ }\end{array}$ & $\begin{array}{c}\text { „suche ei } \\
\text { Interniste }\end{array}$ \\
\hline $0 \%$ & $0 \%$ & $0 \%$ \\
\hline $0 \%$ & $11 \%$ & $0 \%$ \\
\hline $0 \%$ & $11 \%$ & $0 \%$ \\
\hline $100 \%$ & $78 \%$ & $100 \%$ \\
\hline 6 & 3,8 & 4,8 \\
\hline 14 & 18 & 15 \\
\hline 14 & 18 & 15 \\
\hline
\end{tabular}

\begin{tabular}{|l|l|}
\hline $0 \%$ \\
\hline $0 \%$ \\
\hline $10 \%$ \\
\hline $90 \%$ \\
\hline 4,5 \\
\hline 10 \\
\hline 10 \\
\hline
\end{tabular}

me Ereignisse in der Lebensgeschichte des Pseudopatienten wurden so dargestellt, wie sie sich tatsächlich zugetragen hatten. Die Beziehungen zu Eltern und Geschwistern, zu Ehepartner und Kindern, zu Menschen am Arbeitsplatz und in der Schule [...] wurden so beschrieben, wie sie waren; Frustrationen und Ärgernisse ebenso wie Erlebnisse von Glück und Zufriedenheit. " 5 Er kommentiert dieses Vorgehen anschließend kurz so: „Diese wahrheitsgemäße Darstellung der Tatsachen ist von großer Bedeutung, denn sie erhöht die Wahrscheinlichkeit, dass die Probanden als gesund kategorisiert werden, da keine ihrer Angaben zur Vorgeschichte oder ihrer aktuellen Verhaltensweisen in irgendeiner Hinsicht ernsthaft pathologisch waren" [25]. ${ }^{6}$

Nach der Aufnahme berichteten die Pseudopatienten nicht mehr über ihre Stimmen und versuchten, sich normal zu verhalten, mit Ausnahme der Tatsache, dass sie erstens anfangs (verständlicherweise) nervös und ängstlich waren und zweitens über den gesamten Verlauf hinweg schriftliche Notizen zum Verhalten von Ärzten und Pflegepersonal machten. Dies fand - entgegen anfänglicher Befürchtungen - kaum Beachtung. Sie unterhielten sich mit anderen Patienten, befolgten die Anweisungen des Pflegepersonals und berichteten, dass es ihnen gut ging, wenn sie gefragt

5 "After calling the hospital for an appointment, the pseudopatient arrived at the admissions office complaining that he had been hearing voices. Asked what the voices said, he replied that they were often unclear, but as far as he could tell they said 'empty,' 'hollow,' and 'thud'. [...] Beyond alleging the symptoms and falsifying name, vocation, and employment, no further alterations of person, history, or circumstances were made. The significant events of the pseudopatient's life history were presented as they had actually occurred. Relationships with parents and siblings, with spouse and children, with people at work and in school [...] were described as they were or had been. Frustrations and upsets were described along with joys and satisfactions."

6 „These facts are important to remember. If anything, they strongly biased the subsequent results in favor of detecting sanity, since none of their histories or current behaviors were seriously pathological in any way." wurden. Stimmen hörten sie nicht, gaben sie wahrheitsgemäß an. Bei der Aufnahme war, wie allgemein üblich, nicht klar, wann die Pseudopatienten entlassen werden würden. Dies lag an ihnen, und da alle rasch wieder raus wollten, waren sie hoch motiviert, alles daran zu setzen und allem zu entsprechen, was die Entlassung begünstigte. Hierzu bemerkt Rosenhan: „Ihr Verhalten war in keiner Weise störend, wie die Pflegeberichte bestätigen, die bei den meisten Patienten eingeholt wurden. Diese Berichte ergaben immer wieder, dass die Patienten, freundlich ' und ,kooperativ ' waren und ,keine abnormalen Anzeichen ' vorlagen “ [25]. ${ }^{7}$

Neben den Daten in > Tab. 1 gibt die Studie empirisch nicht viel her: Es wurde noch während der ersten 3 von 12 Hospitalisierungen gefunden, dass 35 von 118 Mitpatienten die Pseudopatienten als gesund erkannt hatten, wofür als Beispiel die folgende Bemerkung eines Mitpatienten zu einem Pseudopatienten angeführt wird: „Sie sind nicht verrückt. Sie sind Journalist oder Professor (bezieht sich dabei auf das ständige Aufschreiben von Notizen). Sie überprüfen das Krankenhaus “ [25]. ${ }^{8}$ Weiterhin wurde in 4 Krankenhäusern, in denen das Personal in einem Glaskasten auf den Stationen arbeitete, gemessen, wie lange und wie oft Patientenkontakt stattfand: Das Pflegepersonal verbrachte 11,3\% (Bereich: $3 \%$ bis $52 \%$ ) der Arbeitszeit außerhalb des Glaskastens (also entweder mit den Patienten oder mit dem Aufräumen von Wäsche oder anderen Aktivitäten). Pro Schicht kamen die Pflegekräfte im Mittel 11,5-mal (Bereich: 4- bis 39-mal) aus dem Glaskasten (einschließlich der Gelegenheiten, wo sie die Station ganz verließen). Die Ärzte kamen im Mittel 6,7-mal pro Tag aus dem Glaskasten (Bereich: 1- bis 17mal). Drittens wird noch über eine weitere Statistik berichtet: „Der

7 „That their behavior was in no way disruptive is confirmed by nursing reports, which have been obtained in most of the patients. These reports uniformly indicated that the patients were 'friendly', 'cooperative', and 'exhibited no abnormal indications'. “

8 „You're not crazy. You're a journalist, or a professor [referring to the continual note taking]. You're checking up on the hospital.“ 
durchschnittliche tägliche Kontakt mit Psychiatern, Psychologen, Assistenzärzten und Ärzten lag zwischen 3,9 und 25,1 Minuten, mit einem Gesamtmittelwert von 6,8 Minuten (6 Pseudopatienten mit 129 Tagen Krankenhausaufenthalt) “ [25]. ${ }^{9}$ Bemerkenswert ist schließlich noch, dass den Pseudopatienten fast 2100 Tabletten ausgehändigt worden waren, die sie mit 2 Ausnahmen alle entweder in ihren Taschen oder unter der Zunge versteckt hatten, um sie kurze Zeit später in der Toilette zu entsorgen (wie viele der anderen, wirklichen Patienten auch, wie man von Rosenhan erfährt).

Der überwiegende Teil des Textes beschäftigt sich nicht mit Daten ${ }^{10}$, sondern mit einer Fundamentalkritik der Institution „Psychiatrisches Krankenhaus“, die es abzuschaffen gelte, da sie zu Gefühlen der Machtlosigkeit und Depersonalisation sowie zur sehr schädlichen (und nicht zu korrigierenden) Etikettierung von Patienten und damit nicht zu deren Gesundung beitrage. Eigentlich gesunde Menschen würden erst durch die Etikettierung mittels einer nicht validen Diagnose zu psychisch Kranken gemacht, die dann durch ein grausames und unmenschliches System nur noch verwaltet würden.

\section{Der Betroffene (M. Spitzer)}

10 Jahre später begann ich im Herbst 1983 meine psychiatrische Weiterbildung als Assistenzarzt an der Psychiatrischen Universitätsklinik in Freiburg. Schon davor hatte ich während der höheren Semester meines Psychologie-Studiums (von 1978 bis 1984) den „Labeling-Ansatz“ („Labeling approach“) kennen gelernt und Arbeiten von Sarbin und Mancuso, Goffman und nicht zuletzt auch die von Rosenhan gelesen. Wie bis heute üblich, begann ich meine Assistenzarztzeit in der Psychiatrie auf einer Akutstation, wo man sehr viele sehr kranke Menschen sieht. Dennoch ließ mich der Etikettierungsansatz nicht los, denn ich konnte nach entsprechenden Erfahrungen (die man in der Psychologie als Student oder Professor einfach nicht hat) nicht glauben, wie ein solcher Unsinn Eingang in den wissenschaftlichen Diskurs hatte finden können. Meine Patienten waren definitiv wirklich krank, egal ob ich die Krankheit „Hitzliputzli“ oder „Schizophrenie“ (Bewusstseinsspaltung) nennen würde, und ihr Befinden wurde durch den Aufenthalt in der Klinik meistens tatsächlich gebessert, auch wenn wir noch praktisch nichts darüber wussten, wie und warum unsere Behandlung

9 „Average daily contact with psychiatrists, psychologists, residents, and physicians combined ranged from 3.9 to 25.1 minutes, with an overall mean 6.8 (six pseudopatients over a total of 129 days of hospitalization).“

10 Mit einer Ausnahme: In zwei kurzen Absätzen wird in der Arbeit von Rosenhan ([25], S. 252) noch über ein zweites Experiment berichtet, das durchgeführt worden war, weil die Verantwortlichen einer psychiatrischen Uni-Klinik nach Bekanntwerden der Ergebnisse des beschriebenen Experiments behauptet hatten, dass solche Fehldiagnosen in ihrem Hause nicht vorkommen würden. Rosenhan teilte ihnen mit, er würde innerhalb von drei Monaten einige Pseudopatienten zu ihnen schicken. Die Klinik solle ihrerseits alle Patienten nach ihrer Wahrscheinlichkeit, Pseudopatienten zu sein, einstufen. Während dieser 3 Monate wurden 193 Patienten aufgenommen, von denen 41 von mindestens einem Mitglied des Personals für Pseudopatienten gehalten wurden, 19 davon von einem Psychiater und einem anderen Personalmitglied. Tatsächlich hatte Rosenhan keinen einzigen Pseudopatienten geschickt. wirkte. Entscheidend war (wie in der gesamten Medizin immer), dass sie wirkte.

Die von Rosenhan in den US-Kliniken beschriebenen Zustände konnte ich zunächst ebenfalls nicht glauben (ich lernte erst später während meiner Forschungsaufenthalte in den USA, dass sie wahrscheinlich nicht sehr übertrieben dargestellt worden waren ${ }^{11}$ ). In der Freiburger Klinik jedenfalls war man um Menschlichkeit und Empathie sehr bemüht; der Chef hatte die Einheitskleidung („Sträflingsanzüge“) für Patienten schon lange abgeschafft. Das Pflegepersonal, die Kotherapeuten, Psychologen, Sozialarbeiter und Ärzte gaben sich große Mühe. Aus meiner heutigen Sicht kann man dies daran ermessen, dass die damals herrschenden „Schulenstreitereien“ (zwischen Psychoanalytikern und Verhaltenstherapeuten, Psychologen und biologisch orientierten Psychiatern, Psychopharmakologen und Sozialpsychiatern) bei Besprechungen über einzelne Patienten immer wieder aufflackerten: Man nahm das ernst und wollte es wirklich wissen. Um konkrete „Lösungen“ für den jeweiligen Fall wurde hart gerungen, die grundlegenden Unterschiede im Denkansatz der Beteiligten blieben jedoch bestehen. Immerhin war man aber meist höflich genug, um dem anderen wenigstens zuzuhören. Mir persönlich wurde als „blutiger Anfänger" in dieser noch recht jungen Lebensphase (25 bis 30 Jahre) klar, dass mein Fachgebiet erstens unglaublich spannend ist und daher zweitens viele Änderungen und Umbrüche in den nächsten Jahren und Jahrzehnten zu erwarten sind.

\section{Der Wahrheitssucher (R. Spitzer)}

Ich war froh, als ich einen Aufsatz meines Namensvetters Robert Spitzer (22.5.1932-25.12.2015) ${ }^{12}$ in den Archives of General Psychiatry fand [35], in dem er sehr klar und deutlich zu Rosenhan Stellung bezog: Wenn ein Patient in eine orthopädische Klinik geht und über Rückenschmerzen klagt, dann würde man bei ihm eine Lumbago (zu Deutsch: „Rückenschmerzen“) diagnostizieren, und wenn man dann nichts weiter findet und er seine Schmerzen nicht mehr hat, würde man ihn mit der Diagnose „Lumbago, remittiert“ entlassen. - Egal, ob er gelogen hat oder nicht. Was folgt daraus für die Validität orthopädischer Diagnosen? - Nichts! Später las ich seine ein Jahr früher publizierte erste, sehr beißende Kritik an Rosenhan: „Entsprechend der Entlassung seiner Pseudopatienten unter der Diagnose ,Schizophrenie in Remission', führt eine sorgfältige Untersuchung der Methoden, Ergebnisse und Schlussfolgerungen dieser Studie zur Diagnose ,Logik in Remission'. [...] Als Professor für Rechtswissenschaft und Psychologie weiß Rosenhan,

11 Wenn man auswählen könnte, in welchem Land der Welt man den ersten Schub seiner Schizophrenie erleben kann, würde ich Deutschland dringend empfehlen. In den USA gehört offenbar eine gründliche Ausschlussdiagnostik - mindestens mittels Bildgebung, EEG und Liquordiagnostik - nicht zum allgemeinen Prozedere. Nur wer Geld hat, bekommt ein MR und eine Lumbalpunktion, sodass offenbar noch immer Enzephalitiden mit psychotischer Symptomatik als Schizophrenie fehldiagnostiziert und damit auch insuffizient behandelt werden [4].

12 Ich lernte Robert Spitzer anlässlich meiner Besuche der jährlichen Treffen der US-Psychiater kennen und konnte mehrfach mit ihm sprechen. So erfuhr ich, dass er Vorfahren in Schlesien hat und wir damit - möglicherweise und ganz weit entfernt - verwandt sind, denn mein Vater stammte auch aus Schlesien. 
dass die Begriffe, gesund' (sane) und,geisteskrank' (insane) juristische und nicht psychiatrische Begriffe sind. Er weiß, dass kein Psychiater die Diagnose, geistige Gesundheit' oder ,Geisteskrankheit' stellt und dass die wahre Bedeutung dieser Begriffe, die von Bundesstaat zu Bundesstaat unterschiedlich ist, sich auf die Unfähigkeit eines Menschen bezieht, zwischen Recht und Unrecht zu unterscheiden - ein Thema, das für diese Studie völlig irrelevant ist“ [34]. ${ }^{13}$ Aufgrund ihrer logischen Klarheit ist die kritische Auseinandersetzung von Spitzer mit Rosenhan sehr lesenswert. Aus meiner Sicht sollten beide Arbeiten hierzu [34, 35], wie auch die Arbeit von Rosenhan selbst und das Buch von Cahalan) zur Pflichtlektüre während der Ausbildung zum Psychiater gehören. Als damaliger Psychiatrie-Anfänger habe ich sehr viel von deren Lektüre profitiert. „Dieser Robert Spitzer ist ein großartiger Psychiater, ein Detektiv, der mit klarem Kopf die Wahrheit aufklärt wie Sherlock Holmes" - dachte ich damals.

\section{Das DSM-III}

Etwa zur gleichen Zeit schwappte aus den USA ein völlig neuer Ansatz für psychiatrische Diagnosen nach Deutschland: Das DSM-III (Diagnostic and Statistical Manual of Mental Disorders) war 1980 in den USA erschienen und kam in deutscher Übersetzung 1984 heraus. Das dicke Buch kostete 144 Deutsche Mark und war für eine Weile das teuerste Buch in meinem Bücherregal. Bei diesem neuen Leitfaden psychischer Störungen handelte es sich um die dritte Auflage eines seit 1952 in den USA verwendeten Klassifikationssystems für Erkrankungen im Bereich der Psychiatrie ( $\triangleright$ Tab. 2).

Was als Heftchen mit Ringbindung (eine Reaktion auf die desolate Situation der Psychiatrie und die vielen psychisch kranken ehemaligen Soldaten nach dem zweiten Weltkrieg) begann (DSM-I mit 106 Diagnosen auf 130 Seiten), wurde als nahezu wörtliche Abschrift der ICD-8 (des damaligen Klassifikationssystems der Weltgesundheitsorganisation WHO) mit psychoanalytischen Begleittexten 1968 in zweiter Auflage weitergeführt (DSM-II mit 182 Diagnosen auf 134 Seiten). In deren 6. Auflage aus dem Jahr 1974 wurde Homosexualität als psychiatrische Diagnose nicht mehr aufgeführt. Auch diese 2. Auflage des DSM fand kaum Interesse, weder bei Wissenschaftlern noch bei Klinikern, worauf der Begründer und wesentliche Motor der 3. Auflage, mein Namensvetter Robert Spitzer, beispielsweise mit folgender Anekdote hinwies [36]. Der bekannte Psychoanalytiker Dr. Irving Bieber wurde im Jahr 1973 - im gleichen Jahr war auch Rosenhans Studie erschienen - gefragt: „Hast du die schrecklichen Neuigkeiten gehört? Sie nehmen Homosexualität aus den künftigen Nachdrucken des DSM-II heraus“, Seine Antwort: „Was ist DSM-II?“

Das DSM-III war für einige Zeit meine wichtigste Lektüre und begeisterte mich zunächst sehr. Kein anderer als Robert Spitzer hatte

13 Im englischen Original: Just as his pseudopatients were discharged as 'schizophrenia in remission', so a careful examination of this study's methods, results and conclusions leads tot he diagnosis of 'logic in remission'. Rosenhan, a professor of law and psychology, knows that the terms 'sane' and 'insane' are legal, not psychiatric, concepts. He knows that no psychiatrist makes a diagnosis of 'sanity' or 'insanity' and that the true meaning of these terms, which varies from state to state, involves the inability to appreciate right from wrong-an issue that is totally irrelevant to this study.
- Tab. 2 Übersicht zur Geschichte des Diagnostic and Statistical Manual of Mental Disorders (DSM) der US-amerikanischen Psychiatrischen Vereinigung (American Psychiatric Association, abgekürzt APA1). Die Daten hierzu finden sich über viele Quellen verstreut, wobei diese oft nicht übereinstimmen. Die hier abgedruckten Daten wurden den beiden Einträgen zum DSM in der deutschen und amerikanischen Wikipedia entnommen.

\begin{tabular}{|c|c|c|c|c|}
\hline Version & $\begin{array}{l}\text { Erschei- } \\
\text { nungsjahr } \\
\text { (USA) }\end{array}$ & $\begin{array}{l}\text { Seiten- } \\
\text { zahl }\end{array}$ & $\begin{array}{l}\text { Anzahl } \\
\text { der Diag- } \\
\text { nosen }\end{array}$ & $\begin{array}{l}\text { Erscheinungs- } \\
\text { jahr deutsche } \\
\text { Übersetzung }\end{array}$ \\
\hline DSM-I & 1952 & 130 & 106 & - \\
\hline DSM-II & 1968 & 134 & 182 & - \\
\hline DSM-III & 1980 & 494 & 265 & 1984 \\
\hline DSM-IIIR* & 1987 & 567 & 292 & 1989 \\
\hline DSM-IV & 1994 & 886 & 410 & 1996 \\
\hline $\begin{array}{l}\text { DSM- } \\
\text { IVTR }^{* *}\end{array}$ & 2000 & 943 & 410 & 2003 \\
\hline DSM $-5^{* * *}$ & 2013 & 947 & 374 & 2015 \\
\hline \multicolumn{5}{|c|}{$\begin{array}{l}{ }^{*} \text { Revision; }{ }^{* *} \text { Text Revision; }{ }^{* * *} \text { man stieg auf arabische Zahlen um, } \\
\text { damit - analog zu Computersoftware - künftig kleinere Updates z. B. } \\
\text { als „Version 5.1“ und größere als „Version 6.0“ bezeichnet werden } \\
\text { können. }\end{array}$} \\
\hline
\end{tabular}

die Herkulesaufgabe auf sich genommen, einmal klar zu sagen, was eine psychische Störung ist, und dann ebenso klar zu sagen, was genau bei einer bestimmten Störung an ganz bestimmten Symptomen, d. h. erfragbaren Erlebnissen und beobachtbaren Verhaltensweisen, vorliegen muss, damit die betreffende psychische Störung diagnostiziert werden kann. Man sprach von „diagnostischen Kriterien“ oder „Diagnosekriterien“, und obgleich sie oft nur das klar auszusprechen schienen, was man ohnehin im Kopfe hatte, wenn man die entsprechenden Diagnosen stellte, empfand ich das Ganze als einen sehr befreienden Segen. Denn plötzlich schien klar, worüber man redete, und jeder konnte nachlesen, was man meinte.

So kann ich mich beispielsweise noch gut erinnern, wie ich als Anfänger die Diagnose von Persönlichkeitsstörungen nicht mochte: Da wurde über „tiefe Strukturen und deren Schäden“, „frühe“ Störungen, „schwere Verwerfungen“ oder „dysfunktionale Beziehungsgestaltungsmuster" geredet und mein Eindruck war, dass jeder sagen durfte, was er wollte, wenn man Professor war oder „klinisch sehr erfahren “. Ich war damals weder das eine noch das andere. Aber ich war froh, dass es im DSM-III Kriterien für „Achse-II-Diagnosen“ ${ }^{14}$ gab, die bei genauer Kenntnis des einzelnen Patienten,

14 Im DSM-III wurde die Unterscheidung zwischen „akuten Krankheiten“ (Achse I) und „chronischen Persönlichkeitsstörungen“ sowie Intelligenzminderung (Achse II) eingeführt, drei weitere Achsen betrafen medizinische Krankheiten bzw. organische Befunde (Achse III), psychosoziale Belastungsfaktoren (Achse IV) und das globale Funktionsniveau (Achse V). Mit DSM-III und DSM-IV (also von 1980 bis 2013) bestand damit das Ziel der psychiatrischen Diagnostik in einer umfassenden Beurteilung des Patienten im Sinne des biopsychosozialen Krankheitsmodells, welches für nahezu ein halbes Jahrhundert in der Psychiatrie galt [9]. Was sich als durchaus praktisch erwies, wurde mit dem DSM-5 jedoch wieder abgeschafft $[10,19]$. 
dessen Lebensgeschichte und dessen Erfahrungen, fast so einfach zu stellen waren wie die einer Schizophrenie, Depression oder Alkoholsucht. Angeregt durch die in den Archives publizierten Arbeiten von Spitzer und Mitarbeitern [37] über die Borderline-Persönlichkeitsstörung sowie von Widiger und Mitarbeitern [39] über die Reliabilität der DSM-III-Diagnosen entwarf ich sogar einen Fragebogen, der die DSM-III-Kriterien für alle Persönlichkeitsstörungen in zufälliger (sprich: durcheinandergewürfelter) Reihenfolge enthielt und Auswerteschablonen (aus Pappe), um dann anhand der Angaben des Patienten - und ohne jegliche Hermeneutik (d. h. Vorannahmen und damit mögliche Halo-Effekte) - Persönlichkeitsstörungen untersuchen zu können. ${ }^{15}$ Der Versuch scheiterte, was mich nicht daran hinderte, mich weiter mit den Grundlagen meines Fachs, der Psychopathologie, zu beschäftigen.

Robert Spitzer hatte mit Blick auf die Diagnosekriterien des DSM-III allgemein und die Diagnose der Schizophrenie im Besonderen damals immer wieder geäußert: „Ich weiß nicht, was Schizophrenie ist“ und wandelte damit auf den Spuren der deutschen Psychopathologie von Karl Jaspers und Kurz Schneider: Das Motto von Jaspers war: „Es sollte klar werden, was man weiß, wie man es weiß und was man nicht weiß“; und Kurt Schneider ist für seinen Ausspruch bekannt, dass dann, wenn Erstrangsymptome vorliegen, er „in aller Bescheidenheit von Schizophrenie“ spreche. Spitzer bezog sich in seinen Schriften auf Schneiders Erstrangsymptome, war sich also über diese Tradition seines Tuns im Klaren. Er konnte damals allerdings nicht wissen, dass sein Optimismus nicht ganz so berechtigt war, wie er bei Kurt Schneider lesen konnte.

\section{Übersetzungsfehler mit Folgen}

Dies liegt an folgendem Umstand: Als es mich in den Jahren 1989/90 aufgrund eines Chefwechsels in Freiburg und einer Reihe glücklicher Umstände an die Harvard Universität verschlug, lehrte ich dort Psychopathologie. Dies brachte die Aufgabe mit sich, für die Lektüre im Seminar englische Übersetzungen der deutschen Texte aufzuspüren und zu besorgen, was sich als schwieriger herausstellte als gedacht. Die englische Version der Allgemeinen Psychopathologie von Karl Jaspers konnte ich zwar käuflich erwerben, sie war jedoch aufgrund einer miserablen Übersetzung völlig unbrauchbar [32]. Und an der berühmten Widener Library auf dem Campus (eine der größten Bibliotheken der Welt) konnte man zwar meine im Jahr 1985 als Buch erschienene philosophische Dissertation (was mich sehr erstaunte) ausleihen, wie sich herausstellte,

15 Unerfahren wie ich damals war, bat ich auch meine Kollegen, diesen Fragebogen für mich auszufüllen. Obwohl mein damaliger Chef, Prof. Dr. Rudolf Degkwitz, mich in meinen Bemühungen unterstützte, war dies zum Scheitern verurteilt, weil ich Arbeit generierte, die viele meiner Kollegen (vor allem die klinisch erfahrenen) für unnötig oder gar unsinnig hielten. Sie hatten Recht. Wie meine Auswertungen der von mir selbst fleißig ausgefüllten Bögen (beidseitig bedruckt in DIN-A-3 Format, denn es waren nicht wenige Kriterien) sehr bald zeigten, gab es entgegen meinen Erwartungen keine Symptom-Cluster, die einzelne Persönlichkeitsstörungen voneinander abgegrenzt hätten. Fast jeder Patient erfüllte ein paar Kriterien, so dass ich heute sagen würde, man hätte damals bei sehr vielen Patienten die Diagnose „Persönlichkeitsstörung, gemischt" stellen können. Das war wissenschaftlich enttäuschend und praktisch nicht zielführend. Und so blieb es bei diesem Versuch. gab es jedoch die englische Übersetzung von Kurt Schneiders Büchlein „Klinische Psychopathologie“ aus dem Jahr 1959, die im gleichen Jahr in englischer Übersetzung erschienen war, im gesamten Großraum von Boston (mit seinen damals 32 Universitäten) nicht. Eine Studentin, deren Mutter an der US Library of Congress arbeitete (die damals größte Bibliothek der Welt) konnte mir das Buch schließlich per Fernleihe besorgen. Dort fand ich einen offenbar folgenschweren Übersetzungsfehler: Aus „Bescheidenheit“ wurde in der englischen Übersetzung „Entschiedenheit“ [32]. ${ }^{16}$ Verschwunden war in der englischen Übersetzung die methodisch reflektierte Bescheidenheit. Sie wurde ersetzt durch „Entschiedenheit“, was also „modesty“ hätte lauten sollen, wurde zu „decisiveness“. Über den Grund dieses Fehlers kann man nur Vermutungen anstellen; ich gehe davon aus, dass der Gleichklang von Bescheidenheit und Entschiedenheit eine Rolle spielte.

Man könnte dies als harmlosen Übersetzungsfehler abtun, hätte er nicht so unglaublich gut in die amerikanische Psychiatrielandschaft gepasst: Ausgehend von dem Bleulerschen Krankheitsbegriff mit seinen eher vage definierten Grundsymptomen - im Englischen meistens „unter the classic 4A“ bekannt: Gestörte Assoziation, gestörter Affekt, Ambivalenz (gestörter Wille) und Autismus (gestörtes Sozialverhalten) - herrschte in den USA ein sehr breiter Schizophreniebegriff, was dazu geführt hatte, dass die Diagnose Schizophrenie damals die mit Abstand häufigste psychiatrische Diagnose war. Entstanden durch eine Vermischung des Bleulerschen Konzepts mit psychoanalytischer Terminologie, was definitiv nicht zur Klarheit beigetragen hatte, führte dies in der Psychiatrie in den USA zu dem unhaltbaren Zustand, dass der größte Teil der Patienten (nach Daten aus New York etwa $85 \%$ aller Aufnahmen) nicht nur die gleiche Diagnose erhielt, sondern zudem eine Diagnose erhielten, die äußerst vage definiert war. In dieser Situation musste die „Entschiedenheit“ Kurt Schneiders vielen wie ein Segen vorkommen: Hier war jemand, der klar und eindeutig ohne psychodynamische Schnörkel oder Soziologenkauderwelsch sagte, wie man eine Schizophrenie diagnostiziert. Man stellt bestimmte Symptome fest und wenn diese vorliegen, dann stellt man die Diagnose. Mit Entschiedenheit! Das musste den pragmatischen Amerikanern einfach gefallen!

So wundert es nicht, dass gerade in diesem Land der einfachen und schnellen Lösungen Kurt Schneider immer bekannter und beliebter wurde (Klerman et al. 1984. Als dann noch Anfang der 70er Jahre die Peinlichkeit ans Licht kam, dass die britischen Kollegen mit dem Begriff der Schizophrenie weitaus vorsichtiger umgingen als die Amerikaner [17], war für jedermann klar ersichtlich, dass hier Abhilfe geschaffen werden musste. In den Jahren danach wurden daher die Research Diagnostic Criteria (RDC) entwickelt [33], auf denen letztlich die 1980 publizierten Kriterien im DSM-III basierten.

16 Zumindest den älteren deutschen Psychiatern dürfte bekannt sein, dass Kurt Schneider die Erstrangsymptome vorsichtig formuliert darstellte und sie dann wie folgt kommentiert: „Wo derartige Erlebnisweisen einwandfrei vorliegen und keine körperlichen Grundkrankheiten zu finden sind, sprechen wir klinisch in aller Bescheidenheit von Schizophrenie“ ([27], Hervorhebung durch den Autor). Nicht schlecht staunte ich, als ich in der englischen Übersetzung das Folgende fand: „When any of these modes of experience is undeniably present and no basic somatic illness can be found, we may make the decisive clinical diagnosis of schizophrenia“ ([27], Hervorhebung durch den Autor). 
In den USA wird die Schizophrenie bis heute mit Bezug auf Kurt Schneiders Erstrangsymptome diagnostiziert, - allerdings ohne die europäische bzw. deutsche Bescheidenheit. Das DSM-III wurde mit Pauken und Trompeten angekündigt und durch massives Marketing seitens der American Psychiatric Organisation (APA) in alle Welt vertrieben. Hatte man früher naiverweise immer geglaubt, Wissenschaft würde dadurch fortschreiten, dass sich (zumindest mittel- bis langfristig) die Wahrheit durchsetzt, so wurde man von den amerikanische Psychiatern eines Besseren belehrt: Über diagnostische Kategorien wurde per Handzeichen von selbsternannten Experten abgestimmt und danach wurden diese weltweit sozusagen „verordnet“. Wer sich nicht daran hielt, der hatte Pech: Er konnte seine Arbeit in angesehenen Zeitschriften nicht publizieren oder wurde zumindest versuchsweise daran gehindert. ${ }^{17}$

\section{Brendan Maher und Martha Mitchell}

Während meiner Aufenthalte an der Harvard-Universität war Brendan Maher mein Mentor ( $\mathbf{A}$ bb. 1). Er war Lehrstuhlinhaber für klinische Psychologie mit Spezialgebiet experimentelle Psychopathologie und wurde nicht nur mein Mentor, sondern auch einer meiner besten Freunde.

Brendan arbeitete schon wesentlich länger als ich über Wahn und hatte großes Verständnis für Missverständnisse zwischen Patienten und Ärzten. Er gab dem Phänomen, dass Patienten zuweilen für krank erklärt werden, indem faktische Angaben und vernünftig dargebrachte Meinungen als Wahneinfälle gedeutet werden, den Namen Martha-Mitchell-Effekt [22, 23]. Der Ehemann von Martha Mitchell, John Mitchell, Mitarbeiter im Team von US-Präsident Richard Nixon, vor dessen Wiederwahl im November 1972, wurde während des Wahlkampfes am 7. Juni 1972 für einen Einbruch verantwortlich gemacht, der von 5 Männern im Wahlkampfbüro der gegnerischen Demokraten durchgeführt wurde, das in Washington im Watergate-Gebäudekomplex lag ( $\mathbf{A b b}$. 2). Hierbei sollten Abhöranlagen installiert und Dokumente fotografiert werden. Seine Frau rief daraufhin mehrfach Journalisten an, um auf Ungereimtheiten im Weißen Haus hinzuweisen. Sie ging von einer Verschwörung aus, an welcher der US-Präsident beteiligt sei und zu deren Vertuschung ihr Mann als Sündenbock herhalten solle. Sie wurde am Abend des Einbruchs, an dem sie in einem Hotel in Kalifornien war, vom damaligen Ex-FBI-Agenten und Präsidentenmitarbeiter Stephen King als „politische Gefangene“ in ihrem Hotel festgesetzt, von der Außenwelt isoliert und betäubt. Man glaubte ihr zunächst nicht und erklärte sie für verrückt. Wie sich im Laufe

17 Nicht anders erging es auch mir: Ich hatte Anfang der 1990er-Jahre einen Artikel beim Journal of Abnormal Psychology eingereicht und kam die Kritik zurück, dass ich die Schizophrenie nicht nach DSM diagnostiziert habe. So schrieb ich zurück, dass wir in Heidelberg das Problem, bzw. die „Krankheit“, als dessen Lösung, bzw. „Therapie“, viele das DSM-III betrachtet hatten, niemals gehabt hatten: Der Anteil der an der Heidelberger Klinik als schizophren diagnostizierten Patienten war über 60 Jahre hinweg mit etwa $30 \%$ erstaunlich stabil. Genau dies schrieb ich an den Editor zurück der - zu meiner Überraschung - diesen Klartext verstand und die Arbeit zur Publikation annahm. Vielen anderen erging es wahrscheinlich anders.

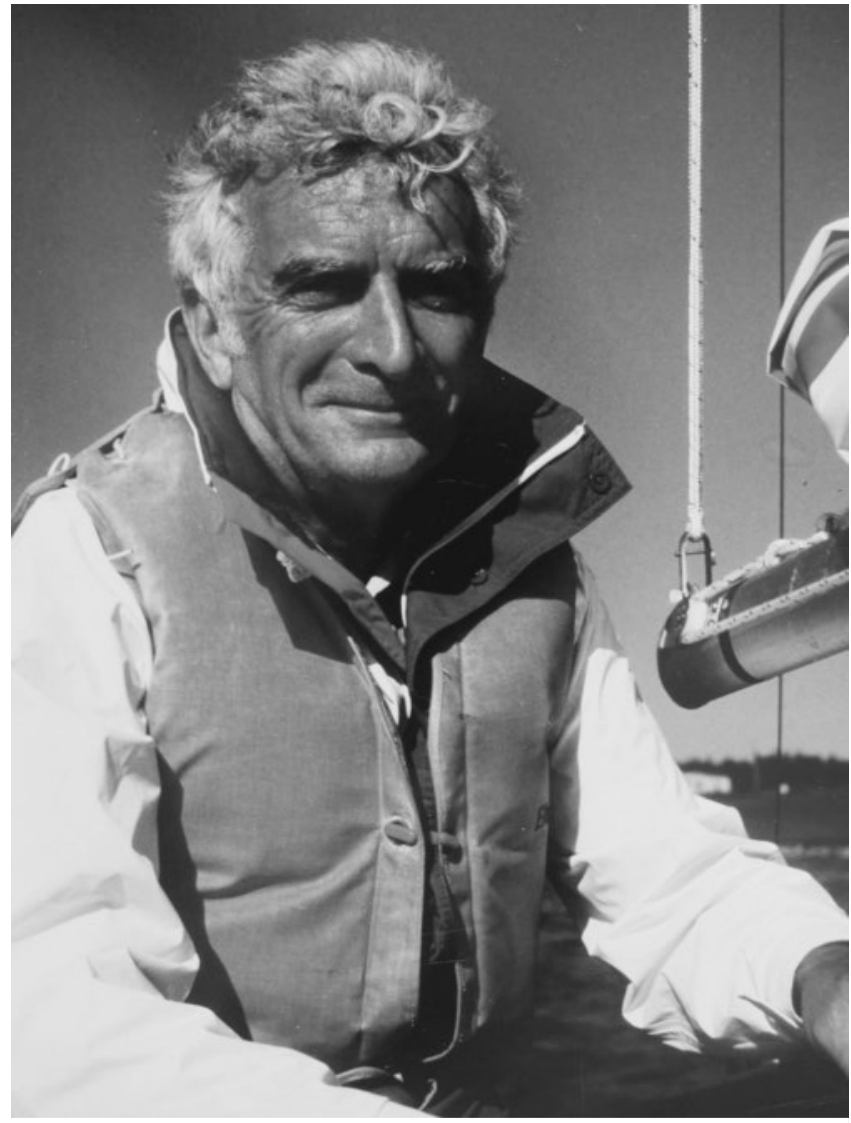

- Abb. 1 Brendan A. Maher (31.10.1924-17.3.2009) war seit seiner Zeit bei der Royal Navy ein begeisterter Segler. Er wurde als Sohn irischer Eltern in England geboren, arbeitete als Minensucher und wurde nach dem Krieg bei einem Unfall bei der Vernichtung von Minen im Hafen von Rotterdam schwer verletzt. Er ging in die USA und promovierte bei dem bekannten Persönlichkeitspsychologen George Kelly im Jahr 1954. Mit seiner Monografie „Principles of Psychopathology: An Experimental Approach“ begründete die experimentelle Psychopathologie und machte sie weithin bekannt $[12,13]$. Im Jahr 1960 ging er als „Lecturer“ an die Harvard University, wo er nach 8 Jahren Aufenthalt an anderen Universitäten im Jahr 1972 Professor für Psychologie wurde. Das Foto entstand während einem der 4 Aufenthalte meiner ganzen Familie auf „seiner“ Insel, auf der außer seinem 200 Jahre alten Ferienhaus kein weiteres Haus stand. (Quelle: ๑ Autor)

des Watergate-Skandals ${ }^{18}$ jedoch herausstellte, hatte sie die Wahrheit gesagt [3], selbst im Hinblick auf ihre kurzfristige Festsetzung mit Waffengewalt und Betäubung durch einen Arzt, wie erst Jahre später öffentlich wurde [38]. Herr King ist seit 2017 US-Botschafter in Tschechien.

18 Zu diesem Skandal führten die Ermittlungen des FBI, die ergaben, dass die Auftraggeber des Watergate-Einbruchs unter engen Mitarbeitern des Präsidenten beziehungsweise seines Wahlkomitees zu suchen waren. Nach Nixons Wiederwahl wurden weitere Verbrechen und Vergehen, die in den Jahren zuvor teils auf direkte Anweisung des Weißen Hauses hin begangen worden waren, aufgedeckt: illegale Wahlspenden, Steuerhinterziehung, Bestechung, Behinderung der Justiz und der - offenbar bis heute übliche - „Verkauf“ von Botschafterposten gegen großzügige Wahlkampfunterstützung. 


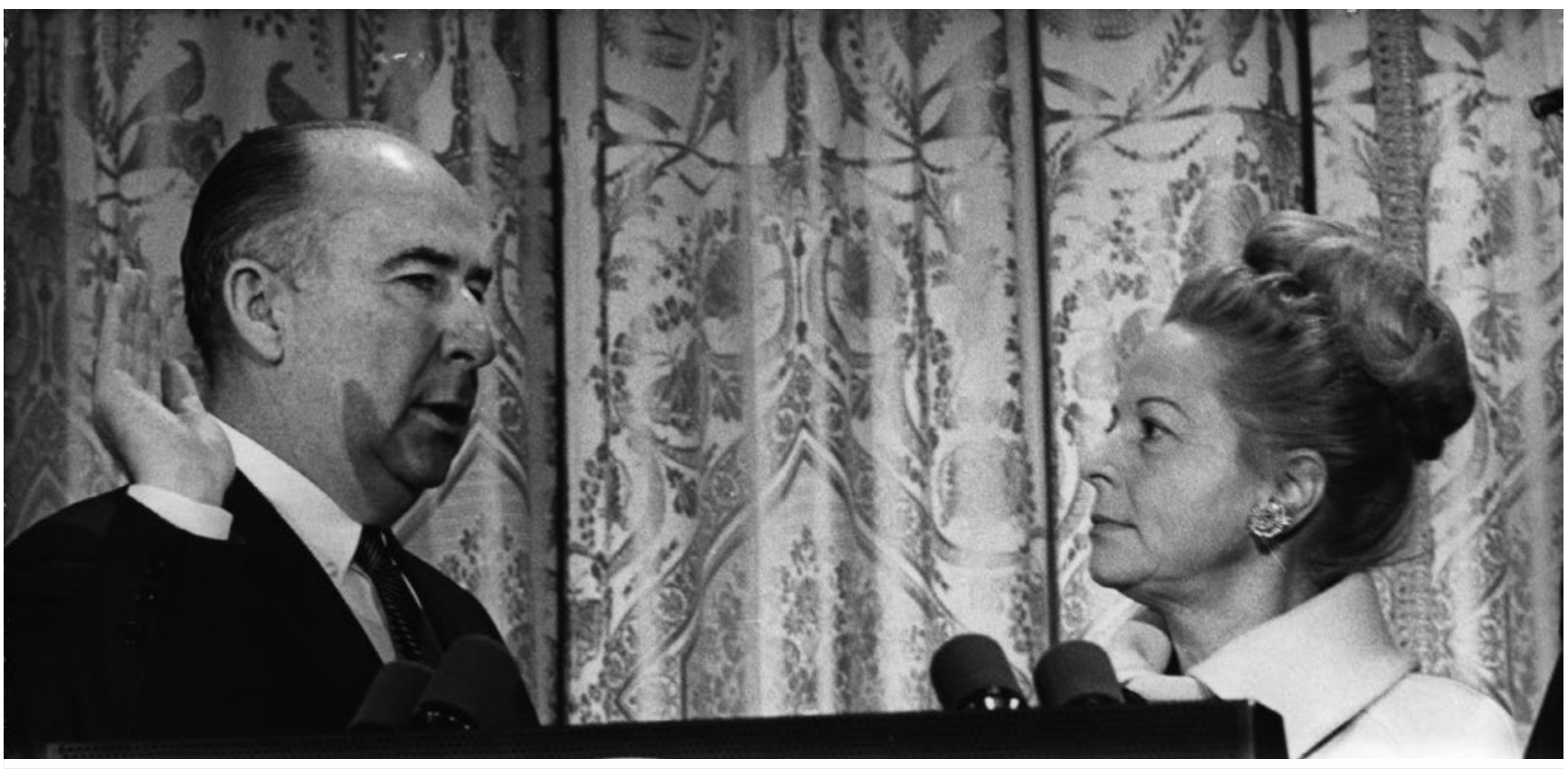

- Abb. 2 Martha und John Mitchell am 11.5.1973 bei der Vereidigung ihres Mannes John als Außenminister der Nixon-Regierung vor dem US-Senat (Quelle: Keystone Press / Alamy Stock Foto).

Irgendwann bei einem der vielen gemeinsamen Gesprächen beim Kaffee erwähnte ich Brendan Maher gegenüber die damals bereits 16 Jahre alte Studie und staunte nicht schlecht über seine Reaktion, die etwa wie folgt lautete: „Oh, Rosenhan - diese Studie hat höchst wahrscheinlich nie stattgefunden. Wir haben das damals heftig diskutiert, hatten ihn zum Vortrag hier ${ }^{19}$, haben nachgefragt, wer die Mitarbeiter waren, die sich in eine psychiatrische Klinik begeben hatten. Er hielt sich damals sehr bedeckt und verwies auf ein Buch, an dem er gerade arbeitete und das alle Details enthielte. Ein solches Buch wurde jedoch nie publiziert, und auch sonst hat er nichts mehr in dieser Richtung getan oder publiziert. Wenn Du mich fragst: Das Ganze ist eine große Lüge.“

\section{Susannah Cahalan: The Great Pretender}

Ich hatte damals einerseits keinen Grund, an Brendans Aussagen über die Rosenhan-Studie zu zweifeln, sprach aber dennoch kaum weiter darüber, und so verblasste das Ganze in meiner Erinnerung. Bis zur Lektüre eines Book-Reviews, der am 31.10.2019 im Fachblatt Nature publiziert worden war [1]. Dort ging es um ein neues Buch der Journalistin Susannah Cahalan mit dem (von der Pop-Gruppe Queen kopierten) Titel „The Great Pretender“.

Thema des gesamten Buchs ist das Experiment von David Rosenhan. Die Autorin litt vor mehr als 10 Jahren an einer Anti-NMDA-Rezeptor Autoimmunenzephalitis, die zunächst als paranoide Schizophrenie verkannt und entsprechend behandelt wurde. Über

19 In einer Fußnote auf der ersten Seite der Arbeit findet sich der Verweis, dass die Daten teilweise bei Colloquien an verschiedenen Universitäten präsentiert worden waren. Als letzte ist die Harvard Universität genannt. Der Vortrag muss also in die Anfangszeit von Brendan Maher als Full Professor für klinische Psychologie gefallen sein. ihre Erfahrungen schrieb sie ihr erstes Buch mit dem Titel „Brain on Fire“ [4, 24]. In einem Gespräch mit Psychiatern erfuhr sie nach der Veröffentlichung ihres Buchs im November 2012 beiläufig von Rosenhans Studie und wollte mehr darüber erfahren. Rosenhan war 9 Monate zuvor verstorben, und so recherchierte Cahalan in den darauffolgenden 6 Jahren mit hoher Intensität und sehr viel Ausdauer. Aus meiner Sicht hat sich ihre Arbeit gelohnt. Das Buch ist nicht zuletzt wegen seines Detailreichtums für alle an Psychiatriegeschichte Interessierte ein Muss und liest sich dazu so spannend wie irgendeine Fiction-Novel von Dan Brown.

Cahalan machte als erstes einen langjährigen Kollegen und Freund von Rosenhan, den Sozialpsychologen Lee Ross, ausfindig. Der übergab ihr nach anfänglichem Zögern den „Nachlass“ von Rosenhan, ein paar Kartons mit Papier. Das Wichtigste, was Cahalan darin fand, waren 8 Kapitel (mehr als 100 Seiten) des Buchs, das nie publiziert worden war. Aber es gab noch mehr: Handschriftliche Aufzeichnungen Rosenhans aus Patientensicht über einen Aufenthalt in einer psychiatrischen Klinik und eine Krankenakte über den Aufenthalt eines Patienten David Lurie in der Psychiatrie im Jahr 1969, bei dem es sich, wie ein Abgleich der Notizen und der Akte zeigte, um die gleiche Person, nämlich Rosenhan selbst, handelte. David Lurie war zugleich der erste im Science-Paper von Rosenhan diskutierte „Pseudopatient“. Drittens gab es Notizen zu weiteren Psychiatrie-Erfahrungen anderer, viertens Hinweise, die Cahalan zu Rosenhans Sohn Jack und seiner langjährigen Bekannten, der klinischen Psychologin Florence Keller führten, sowie fünftens - man glaubt es kaum - Korrespondenz zwischen Rosenhan und Robert Spitzer - den Erzfeinden.

Fassen wir zusammen, was auf den 300 Seiten des Buchs von Cahalan ausführlich beschrieben und penibelst dokumentiert ist: Im Jahr 1969 lehrte Rosenhan noch am Swartmore College in Bundesstaat Pennsylvania an der Ostküste der USA Psychologie. Seine 
Studenten fanden seinen Unterricht zu theoretisch, beschwerten sich und fragten nach Praktika in der Psychiatrie, um klinische Praxis zu gewinnen. Rosenhan schlug ihnen daraufhin vor, nicht als Praktikanten, sondern als Patienten in die Kliniken zu gehen, um einen wirklichen und direkten Eindruck von psychischer Krankheit zu erhalten. Er machte sich dann jedoch zunehmend Sorgen ${ }^{20}$, ob dies gutgehen könne, und ging daher zunächst selbst für 9 Tage in eine nahegelegene psychiatrische Klinik, das Haverford State Hospital, auch „Haverford Hilton“ genannt, weil es 7 Jahre zuvor erst neu gebaut worden war und daher im Vergleich zu anderen großen staatlichen Kliniken moderner, luftiger und vor allem heller war. Wie damals in den USA offenbar üblich, musste er (zusammen mit seiner Frau Mollie) zustimmen, dass er zunächst für unbestimmte Zeit auf eine geschlossene Station aufgenommen wurde. Um sicherzustellen, dass er wirklich aufgenommen werden würde, hatte Rosenhan seine ältesten Klamotten und alte dreckige Schuhe angezogen. Zudem schilderte er nicht nur das Hören von Stimmen, sondern auch, dass er „sensibel für Radiosignale sei und die Gedanken anderer Menschen hören “ könne, und dass er dies schon dadurch versucht hatte zu bekämpfen, indem er „Kupfer über seine Ohren“ gestülpte hatte (die wörtlichen Zitate entstammen wörtlichen Zitaten dessen, was Rosenhan damals gesagt hatte, dokumentiert durch den Aufnahmearzt, Dr. Bartlett, und sind dem Facsimile des Aufnahmebefundes vom 6. Februar 1969 entnommen; [7]). Auch einer der behandelnden Ätzte, Dr. Browning, notierte, dass der Patient „einen Kupfertopf vor sein Ohr gehalten“ habe, um die von ihm empfangenen Signale zu blockieren [7].

Zudem gab er Suizidgedanken an und machte zur Vorgeschichte Angaben, die einen bereits längeren Krankheitsverlauf nahelegten. Dies führte zur Aufnahme unter falschem Namen (David Lurie) und der Diagnose „Schizophrenie, schizoaffektiver Subtyp“, wie aus der Krankenakte der Klinik über Rosenhans Aufenthalt hervorgeht. Sein Arzt, Dr. Bartlett, hatte nicht auf einem stationären Aufenthalt bestanden, da Rosenhan jedoch stationär aufgenommen werden wollte, bestand Bartlett auf der Aufnahme auf der Geschlossenen, wobei er die sehr beunruhigte Ehefrau mit den Worten zu beruhigen versuchte: „Insulin- und Elektroschocks oder andere ähnliche Therapien wenden wir nur nach Rücksprache mit den Angehörigen an" [7]. Mollie dürfte das kaum beruhigt haben. Bartlett war nicht der kalte, distanzierte Psychiater, der sich nicht um die ihm anvertrauten Personen kümmerte (wie von Rosenhan implizit behauptet), sondern das genaue Gegenteil: Nicht zuletzt aufgrund einer schweren Depression, die bei seiner Frau nach Geburt des ersten gemeinsamen Kindes auftrat und zeitlebens fortbestand, war er Advokat der psychisch Kranken, der sich auch noch nach seiner Berentung ehrenamtlich für sie einsetzte.

Auf 23 Seiten beschreibt Cahalan Rosenhans Psychiatrie-Aufenthalt in Kapitel 10 ihres Buchs („Nine days inside a madhouse“) sehr detailreich. Er muss sich miserabel gefühlt haben, schrieb er doch schon am zweiten Tag seines Aufenthaltes über Thomas Szaz, den

20 Er war damit nicht alleine. Auch die Eltern der Studenten, die Rosenhan zur Sicherheit um ihr Einverständnis gebeten hatte, waren nicht begeistert. Eine Mutter schrieb: „Ich erlaube Ihnen hiermit, dass mein Sohn an ihrem verrückten Experiment über die Verrücktheit teilnimmt“ („I hereby give you permission for my son to participate in your insane experiment on insanity“; [7]). vielleicht prominentesten Vertreter der Antipsychiatrie: „Tomas Szaz liegt falsch. Sie [die Patienten] sind wirklich von mir verschieden “ [7]. ${ }^{21}$ An diesem Tag wurde auch seine Diagnose in den „Subtyp: residual“ geändert. „Die Finanzbuchhaltung hat offensichtlich die Leitung der Küche übernommen, “ trägt er am vierten Tag ein. Am fünften Tag findet sich der Rat eines Patienten: „Sag’ nicht, dass es Dir gut geht. Sie werden es Dir nicht glauben. Sage, dass Du noch immer krank bist, aber dass es dir schon besser geht. Das nennt man Einsicht, und sie werden Dich entlassen“.

Er schrieb viel, und so wurde er gefragt: „Was schreiben Sie?“ - „Ein Buch.“ - „Warum schreiben Sie so viel?“. Ein anderer Patient fragte ihn direkt: „Sind Sie ein verdeckter Journalist?“. Am Tag 8 erhielt er tagsüber Probeurlaub nach Hause und am Tag danach wurde er entlassen. Seine Forschungsassistentin Bea Patterson erzählte Cahalan, dass sie „ziemlich schockiert“ war, als sie ihn direkt danach sah. „Diejenigen Studenten in seinem Seminar über klinische Psychologie, die ich interviewen konnte, berichteten mir, dass sich seine Stimmung nach der Rückkehr aus dem Krankenhaus verdunkelt hatte. Er wirkte erniedrigt. Ein Student erinnerte sich daran, dass er gestresst, ausgelaugt und älter aussah als zuvor. Die Studenten wollten mehr von ihm erfahren, aber er weigerte sich, darüber zu sprechen. Eines war nun klar. Sie würden das Experiment nicht weiterführen. Es war vorüber. Erledigt“.

\section{Zwei Pseudopatienten}

Irgendwie schien es irgendwann aber doch weitergegangen zu sein. Denn von den übrigen 7 Pseudopatienten konnte Cahalan durch wahrhafte Detektivarbeit 2 identifizieren und kontaktieren, Rosenhans Graduate-Student und für einige Jahre späteren Mitarbeiter Wilburn (Bill) Underwood, Ph.D., sowie Harry Landow. Underwood hatte ganz offensichtlich eine schreckliche Zeit im Great Asylum for the Insane (später umbenannt in Agnes Landesklinik), eine mit bis zu 4500 Patienten stark überfüllte und personell zugleich unterbesetzte alte heruntergekommene Psychiatrie. Nach seiner Aufnahme (Diagnose: paranoide Schizophrenie) kam Bill in einen Saal mit 20 anderen männlichen Patienten auf einer der Akutstationen. Etwa eine Stunde bevor seine Frau Maryon ihn das erste Mal besuchte, nahm er versehentlich seine Medikamente ein und war danach müde, abwesend und ihm war alles egal. Seine Frau war entsetzt. Nach nur 2 Tagen auf der Akutstation wurde Bill auf eine (halb-)offene Station verlegt, wo es mehr Platz, Sitzgelegenheiten und Ausgang im Freien gab (den Zaun hätte man überspringen können). Nach 9 Tagen wurde Bill entlassen, ohne Diagnose. Angesichts der damals am Agnes-Krankenhaus üblichen Liegezeiten von 130 Tagen war das ein sehr kurzer Aufenthalt. In den Jahren nach seinem Psychiatrie-Aufenthalt hielt er Vorträge darüber, „gab sich aber Mühe, seinem Chef nie die Show zu stehlen“. Die Diskussion des Pseudopatienten Bill ergab folgende neue Erkenntnisse.

21 Die Stelle sei im Original etwas ausführlicher zitiert: „,Tom Szaz is wrong', he wrote, referring to the author of The Myth of Mental Illness. ,They really are different from me.' (Despite being associated with Szaz and the anti-psychiatry movement, Rosenhan complained about being lumped in with him, namely because of their belief that mental illness was not real“ (S. 89). 


\section{On Being Sane in Insane Places: A Supplemental Report}

\author{
The author gives the psychiatric \\ institution a favorable review after \\ spending 19 days as a pseudopa- \\ tient in the psychiatric ward of a \\ large public hospital. He recom- \\ mends stressing the positive as- \\ pects of existing institutions in fu- \\ ture research.
}

\section{Conclusions}

My overall impressions of the hospital are overwhelmingly positive. The powerlessness and depersonalization of patients so strongly emphasized by Rosenhan simply did not exist in this setting. On no occasion did I observe the approach of a patient ignored by staff. In contrast to his findings, average daily contact with doctoral staff was more than an hour. Contact with nurses and attendants was even more extensive.

- Abb. 3 Fotokopie der Zusammenfassung (links) und der Schlussfolgerungen (rechts) aus dem Bericht des neunten Pseudopatienten Harry Lando (aus [18, S. 47, 51]).

- Bei Entlassung gab es nicht „in allen Fällen“ eine Diagnose „Schizophrenie, in Remission“, wie Rosenhan im SciencePaper behauptet.

- In Anmerkung 8 der Arbeit von Rosenhan steht jedoch Folgendes: „[...] a writ of habeas corpus was prepared for each of the pseudopatients and an attorney was kept 'on call' during every hospitalization“. Für Bill gab es jedoch definitiv keine „Habeas Corpus Akte“ und einen „Rechtsanwalt in Rufbereitschaft“, wenn etwas schief gegangen wäre, gab es zu keinem Zeitpunkt. Dies ergaben Cahalans Recherchen bei den entsprechenden Stellen.

- Als Bills Frau zu Besuch kam und ihren Mann intoxikiert vorfand, war Rosenhan schon da (und sie sehr überrascht darüber). Das passt gar nicht zur Darstellung im Science-Paper, dass die Pseudopatienten während ihres Aufenthaltes auf sich gestellt gewesen seien.

- Rosenhans Behauptung, er sage nichts über seine Pseudopatienten, um ihre Anonymität zu wahren, passt nicht dazu, dass er seinen Mitarbeiter Bill Underwood über Jahre hinweg Vorträge über dessen Erfahrungen halten ließ.

Der zweite Pseudopatient, den Cahalan auftreiben und interviewen konnte, war Harry Lando. Der hatte im Jahr 1976 im Fachblatt Professional Psychology seine Sicht der Dinge in einer Arbeit mit dem Titel „On being Sane in Insane Places: A Supplementary Report“ beschrieben: Sein Erlebnisbericht beginnt mit dem Satz: „Ich war der neunte Pseudopatient in der Rosenhan-Studie; und meine Daten wurden in der Originalarbeit nicht berücksichtigt“ [18]. Die erwähnte Endnote Nummer 6 lautet wörtlich wie folgt: „Daten eines neunten Pseudopatienten wurden in diesen Bericht nicht aufgenommen, obwohl Gesundheit nicht erkannt wurde. Aber er hatte Aspekte seiner Biografie, einschließlich seines Familienstandes und möglicher Bekanntschaften, falsch dargestellt. Sein Verhalten im Experiment war damit nicht identisch mit dem der anderen Pseudopatienten“ “ 25]. Fakt ist, dass Rosenhan selbst einige Fakten über sich falsch dargestellt hatte, um aufgenommen zu werden. Warum also schloss er Lando dann aus diesen Gründen aus? Wie Cahalan ausführlich in einem Kapitel über Lando ([6], Kapitel 22: „The Footnote“) beschreibt, hatte dieser in der Psychiatrie sehr positive Erfahrungen gemacht. Diese seien hier anhand zweier Ausschnitte (der Zusammenfassung am Anfang und den Schlussfolgerungen am Ende der Arbeit; Kopien aus dem Original in \ Abb. $\mathbf{3}$ links und rechts) dargestellt.

Mehr gibt es im Grunde nicht zu sagen: Lando fühlte sich in der Psychiatrie gut aufgehoben, war sehr angetan von dem dort arbeitenden Personal und sein Gesamteindruck war „überwältigend positiv“. Das passte Rosenhan ganz offensichtlich nicht ins Konzept, und so wurden die Erfahrungen des neunten Pseudopatienten einfach „aus methodischen Gründen“ nicht weiter erwähnt.

\section{Pseudopatient Rosenhan}

Zurück zu Rosenhan als Pseudopatient. Hier liefern die erhaltenen Dokumente mit Abstand die meisten Informationen, vor allem aber auch eine erhebliche Menge von Ungereimtheiten mit dem, was in der Science-Arbeit nachgelesen werden kann.

- Sein Aufenthalt lag 3 Jahre zurück und war nicht im Rahmen einer experimentellen Studie erfolgt, sondern als eine Art Selbsterfahrung mehr als 3 Jahre zuvor.

- In Science wird beschrieben, wie schon allein die Formulierungen in der Krankenakte „unabsichtlich durch das Personal so verdreht wurden, dass sie der [damals] populären [psychoanalytischen] Theorie der Dynamik einer schizophrenen Reaktion entsprechen."

Cahalan kommentiert angesichts der Gegenüberstellung dessen, was in der Krankenakte steht und dessen, was im Science-Paper steht ( $\triangleright$ Tab. 3), dass es hier definitiv nicht um unbeabsichtige Verfälschungen durch das Personal geht, sondern um beabsichtige Verfälschungen und freie Erfindungen durch den Autor Rosenhan. 
- Tab. 3 Gegenüberstellung der tatsächlichen Krankenakte und dem in Science abgedruckten „Zitat“.

Was in der Krankenakte tatsächlich steht [6, S. 190]

This 39-year-old, white, married, Jewish male was admitted on February 6, 1969 on a 314 commitment. The patient came to the hospital on his own volition and apparently was seeking help. Review of the history reveals that since summer of ' 68 ' the patient has stopped working and has shown a definite social withdrawal. He started to experience auditory hallucinations in November of ' 68 ' and had to resort to some bizarre behavior in order to deal with this experience. When seen in New Case conference on February 11, 1969, the patient was friendly and cooperative, speech was relevant and coherent, and appeared to be of extremely high intelligence. Since being hospitalized he reports complete alleviation of his hallucinatory experiences ...
Was in Science als „Eintrag in die Krankenakte“ steht [25, S. 253]

This white 39 -year-old male ... manifests a long history of considerable ambivalence in close relationships, which begins in early childhood. A warm relationship with his mother cools during his adolescence. A distant relationship to his father is described as becoming very intense. Affective stability is absent. His attempts to control emotionality with his wife and children are punctuated by angry outbursts and, in the case of the children, spankings. And while he says that he has several good friends, one senses considerable ambivalence embedded in those relationships also ...
Auch „ist in der Science-Arbeit weder von Suizidalität noch von Kupfertöpfen die Rede“, [6]).

Im Rahmen der Auseinandersetzungen mit seinem Kritiker Robert Spitzer wollte Rosenhan verhindern, dass Spitzers Kritik publiziert wird und bat mehrere Personen, sich dafür bei Spitzer einzusetzen. Er kommentierte seine Mühen in einem Brief an Spitzer wie folgt: „Sie haben es nun von mir und vom Chef der Klinik (der meine Einweisung arrangierte), dass mein Aufenthalt dort Teil meiner Lehrtätigkeit war und nichts direkt mit Forschung zu tun hatte“ [6]. Dies widerspricht direkt dem Inhalt von $>$ Tab. 3 , d. h. der Tatsache, dass er seinen Aufenthalt durchaus im Science-Paper ausführlicher diskutiert als die Erfahrungen der übrigen Pseudopatienten.

Ein besonders drastisches Beispiel freier Erfindung stellt die Tatsache dar, dass Rosenhan später behauptet hatte, er habe während des Psychiatrie-Aufenthaltes eine Perücke getragen, um nicht erkannt zu werden. In einem Interview, das Cahalan mit einem von Rosenhans Kollegen, Prof. Ervin Staub, geführt hatte, erzählte dieser davon. Sie seien beide nach oben gegangen, wo Rosenhan die Perücke aufbewahrt hatte. „Sie war etwas wild, [die Haare] ein bisschen zu lang. [...] Es war eine interessante Perücke - irgendwie passend für einen Professor “ zitiert Calahan ihren Interviewpartner [6]. Cahalan fand in den Akten jedoch nicht nur den Aufnahmebefund, sondern auch ein Foto von Rosenhan bei der Aufnahme - ohne Perücke. Hinzu kommt zu alldem: Trotz jahrelanger intensiver Suche konnten keine weiteren Pseudopatienten gefunden werden. Cahalan beschreibt in mehreren Kapiteln ihres Buchs einige heiße (und noch ein paar lauwarme) Spuren, die jedoch alle ins Leere führten. Im Jahr 2017 publizierte sie schließlich im Fachblatt Lancet Psychiatry einen Aufruf an die Pseudopatienten von Rosenhan, sich doch bei ihr zu melden [5]:

Sie arbeite gerade an einem Buch über die Studie von Rosenhan. „Ich schreibe, um jeden, der etwas über die Studie weiß, zu erreichen. [Ich möchte herausfinden], wie sie durchgeführt wurde, wer daran beteiligt war, wo die Studie stattfand und wie es dazu kam, dass sie in Science veröffentlicht wurde. Ich halte diese Anfrage bewusst vage, um möglichst viele Menschen zu erreichen. Ich möchte jedoch hinzufügen, dass ich vor allem nach Informationen zu den an der Studie beteiligten Pseudopatienten und Kliniken suche. Ich sammle auch Anekdoten, Erlebnisse und Eindrücke von denjeni- gen, die David Rosenhan persönlich kannten und bin offen für alle Hinweise. "22 Ohne Erfolg.

Aus dieser Tatsache, sowie aus den beiden Befunden, dass in Rosenhans Aufzeichnungen zu keinen weiteren Pseudopatienten Aufzeichnungen zu finden waren, stattdessen jedoch in seinem unfertigen Buch Aufzeichnungen seines Sohnes und anderer Bekannter Rosenhans, die nicht in der Science-Arbeit vorkommen, gleichsam „hineingestopft“ auffindbar waren, drängt sich folgender Gedanke auf. Es sieht so aus, als habe er versucht, das Buch zu schreiben und sich bei der genaueren Schilderung der Pseudopatienten - im Nachhinein, denn es gab sie ja gar nicht - an Berichte anzulehnen, die ihm ohnehin vorlagen.

Rosenhans jüngerer Bruder litt an einer bipolaren Störung, weswegen sich Rosenhan öfter um dessen körperliches und finanzielles Wohl kümmern musste, wie sein Sohn Jack der Autorin mitteilte. Rosenhan wusste also durchaus, dass es psychische Krankheit tatsächlich gibt, d. h. dass sie nicht erst durch „Etikettierung“ durch Psychiater entsteht. Daher wollte er auch nicht mit Thomas Szaz in einen Topf mit den „Antipsychiatern“ geworfen werden. Es konnte ihm mithin in seiner Arbeit nicht um die Abschaffung der Psychiatrie gehen, sondern um die Abschaffung der psychiatrischen Krankenhäuser, wie es sie in den USA damals gab, wie auch weitere Äußerungen Rosenhans in Interviews zeigen. Zum Zeitpunkt der Publikation seiner Arbeit war dies jedoch schon in vollem Gange.

22 „I'm writing to reach anyone with information about the study, namely how it was conducted, who was involved, where the study took place, and how it came to be published in Science. I'm keeping this request purposefully vague as to cast the widest net possible. I will add, though, that l'm primarily searching for any information about the study's so-called pseudopatients, or volunteers, and the hospitals involved in the study. I'm also gathering anecdotes, interactions, and impressions from those who knew David Rosenhan personally. I'm open to all leads." 
Nachdem Rosemary Kennedy ${ }^{23}$, die damals 23-jährige bildhübsche Schwester des späteren amerikanischen Präsidenten John F. Kennedy, durch eine im Jahr 1941 (auf Betreiben ihres Vaters und ohne Wissen der Mutter) durchgeführte Lobotomie zur geistig und körperlich Schwerstbehinderten wurde, hatte ihr älterer Bruder John, der sich erst im Jahr 1960 zu ihr öffentlich bekannte, als Präsident die Initiative ergriffen, um das Los psychisch kranker Menschen zu verbessern. Das Resultat: 10 Jahre später waren die Hälfte aller psychiatrischen Krankenhausbetten in den USA schon nicht mehr da.

\section{Rosenhan und R. Spitzer}

Zum Skurrilsten, was man in Cahalans Buch lesen kann, gehört die Korrespondenz zwischen David Rosenhan und seinem Erzfeind Robert Spitzer. Diese befand sich bei dem von der Autorin gesichteten Nachlass in einem eigenen Ordner mit der Beschriftung „Spitzer, Robert“ [6]. Ein Jahr nach der Publikation von Rosenhans Arbeit - Spitzer war gerade dabei, seine Kritiken zu verfassen - begann er die Korrespondenz mit „Lieber Dave“, was Cahalan zur Bemerkung veranlasst, dass Rosenhan ansonsten nicht mit „Dave“ angesprochen wurde (beide kannten sich ja auch nicht persönlich), weswegen die Anrede sich „eher anfühlt wie ein Ellenbogen an den Rippen als ein Handschlag“ [6]. Spitzer hatte einige Fragen an Rosenhan, dessen Antwortbrief eine „unterschwellige Wut" verrät - und das, obgleich sich beide mit Höflichkeitsfloskeln wie „Yours sincerely“ (Rosenhan) und „Sincerely Yours“ (Spitzer) in ihren wechselseitigen Briefen überboten. Cahalan kommentiert: „Ich stelle mir vor, wie Rosenhan vor ganzen Stapeln von Papieren an seinem Schreibtisch sitzend, den Zeigefinger an seiner Schläfe, mit zunehmend gerötetem Gesicht diese Schreiben las; zugleich stelle ich mir Spitzer vor, der fröhlich lächelnd seine Attacken vor sich hin tippte..." [6]. Der Dialog zwischen beiden wurde zunehmend eisiger, sodass R. Spitzer irgendwann äußerte, man könne nur noch die Hoffnung haben, dass die wechselseitigen Briefe wieder kürzer werden, und Rosenhan danach Briefe geschrieben hat, die „zum Wütendsten gehören, was ich je gesehen habe“, wie Cahalan es zusammenfasst - ohne weitere Zitate anzuführen.

Aber war Robert Spitzer wirklich die wahrheitssuchende Lichtgestalt der Psychiatrie der 1980er-Jahre oder hatte er - wie Sherlock

23 Das Schicksal von Rosemary Kennedy (13. 9.1918-7.1.2005) bewegt einen noch heute und bewirkt Trauer, Wut und Mitleid zugleich: Bei ihrer Geburt war es zu einem Sauerstoffmangel im Gehirn gekommen, weswegen bei ihr eine Entwicklungsverzögerung, eine leichte geistige Retardierung sowie eine Lese-Rechtschreibschwäche vorlagen. „Sie lernte spät laufen, schwamm aber begeistert, tanzte gern und schrieb Tagebücher. Sie nahm rege am sozialen Leben teil und besuchte leidenschaftlich gern Opernaufführungen, Sportveranstaltungen und andere gesellschaftliche Anlässe und wuchs zu einer lebenslustigen Erwachsenen heran“, erfährt man in der Wikipedia. Weil der streng katholische Vater Joseph Kennedy befürchtete, sie könnte schwanger werden, ließ er zu ihrer „Besänftigung“ durch Walter Freeman eine Lobotomie durchführen, deren Folgen katastrophal waren: „Die vom Vater erhoffte Verbesserung blieb aus; stattdessen verschlechterte sich ihr Zustand nach der Operation so sehr, dass sie nur noch kindlich vor sich hinbrabbeln konnte, inkontinent wurde und teilweise auf einen Rollstuhl angewiesen war. Joseph Kennedy, der sich seiner nun schwerbehinderten Tochter schämte, ließ sie in die St.-Coletta-Heilanstalt in Wisconsin einliefern, wo sie den Rest ihres Lebens verbrachte. Er besuchte sie nie und geriet in Rage, wenn man ihn auf sie ansprach “ [41].
Holmes - auch dunkle Seiten? Der genannte, Rosenhan im Jahr 1969 „,behandelnde“ Arzt, Dr. Frank Bartlett war verständlicherweise beleidigt darüber, wie Rosenhan ihn in seiner Arbeit portraitierte. Wie aus der Korrespondenz zwischen Rosenhan und Spitzer hervorgeht [6], verschickte Bartlett daher die Krankenakte „Rosenhan“ an den Psychiater Robert Woodruff, dessen Kritik an Rosenhans Arbeit er gelesen hatte. Dieser schickte sie an Spitzer. Der wusste also, dass es sich beim Patienten David Lurie tatsächlich um David Rosenhan handelte, konnte daher ebenfalls die Akte mit der Science-Arbeit vergleichen und hätte die Rosenhans Lügen spätestens Mitte der 1970er-Jahre ebenso aufdecken können wie Cahalan dies 45 Jahre später dann tatsächlich getan hatte. Warum hat er es dann nicht getan?

Zur Beantwortung dieser Frage zitiert Cahalan aus dem Buch des den Soziologen Andrew Scull mit dem Titel Psychiatry and its Discontents: „Paradoxerweise war die außerordentliche Wirkung von Rosenhans Studie in der Öffentlichkeit wie himmlisches Manna. Denn sie lieferte letztlich den Anstoß für ein Forschungsprojekt, für das er schon seit einiger Zeit geworben hatte, nämlich für die Gründungen eine Arbeitsgruppe der American Psychiatric Association mit dem Auftrag, die Diagnosen in der Psychiatrie neu zu überdenken “ [42]. ${ }^{24}$ Mit anderen Worten: Ohne Rosenhan kein DSMIII - und das war das große Projekt von Spitzer, mit dem er die Psychiatrie der kommenden Jahrzehnte - nicht nur in den USA, sondern weltweit - wesentlich prägte.

Des einen Strandgut war des anderen Schiffbruch: Weil Rosenhan wusste, dass Spitzer wusste, was er wusste, war die Publikation des Buchs sinnlos geworden, denn Spitzer hätte es jederzeit leicht entlarven können. Obgleich Rosenhan 10000 US Dollar vom Verlag Doubleday als Vorschuss bekommen hatte - damals viel Geld - musste er den Plan aufgeben, durch ein paar zusätzliche Lügen seine Science-Publikation zu „stützen“.

\section{Diskussion}

Der Stanford Professor für Psychologie und Recht, David Rosenhan, kam mit seiner im Fachblatt Science im Januar 1973 publizierten Arbeit „On Being Sane in Insane Places“ zu weltweiter Berühmtheit. Die Arbeit gehört nicht nur zu den am häufigsten zitierten Arbeiten im Bereich der Psychiatrie, sondern diese verändert, insbesondere das Los der Patienten: Zwar wurden schon in den 10 Jahren vor Erscheinen der Arbeit etwa $50 \%$ aller psychiatrischen Krankenhausbetten in den USA abgeschafft, aber in den 10 Jahren danach wurde von den verbliebenen Betten nochmals die Hälfte abgeschafft. Zwar mögen sowohl JF Kennedy als auch David Rosenhan gedacht haben, dass das hierdurch eingesparte Geld auf andere Weise zu den bedürftigen Patienten gelangen würde. Bekanntermaßen war dem jedoch nicht so. Dies erwies sich für die praktische Versorgung der wirklich psychisch kranken Menschen als Desaster. Sie endeten auf der Straße und vor allem in Gefängnissen. Was zu Beginn der Psych-

24 „For Spitzer, paradoxically, Rosenhan's study and the extraordinary publicity it received was Manna from heaven. It provided the final impetus for a study he had been agitating to conduct for some time, to set up a task force oft he American Psychiatric Association charged with revamping psychiatry's approach to diagnosis. Das Buch von Scull kann ich sehr empfehlen, weil es die Entwicklung des DSM und die Krise des DSM-5 in seiner jetzigen Form genau beleuchtet. “ 
iatrie als einer ihrer größten Anfangserfolge zu bewerten ist - die Kranken wurden aus den Gefängnissen befreit und nicht mehr als Kriminelle, sondern als krank behandelt, wurde wenn auch mit besten Absichten, innerhalb von 2 Jahrzehnten zunichte gemacht.

Wenn heute deutsche Sozialpsychiater es als Fortschritt betrachten, dass sich die Polizei und das Gefängnis (und nicht der Psychiater auf einer geschlossenen Station) um erregte aggressive psychisch Kranke Menschen in Ausnahmesituationen kümmern mögen [43], sei ihnen entgegengehalten, dass sie aus der Geschichte nichts gelernt haben und ein vollkommen unpraktikables System fordern. Ich möchte nicht mit einer Enzephalitis (wie Cahalan) oder einem Hirntumor zwischen Akutstation und Gefängnis (jeweils mit Blaulicht und Polizeibegleitung) hin- und hergefahren werden, sondern wünsche mir, dass mich ein Kollege im psychiatrischen Akutkrankenhaus mit Worten und Medikamenten beruhigt und ein MRT sowie eine Liquorpunktion durchführt. Kranke Menschen gehören in ein Krankenhaus und nicht ins Gefängnis - das gilt auch und gerade psychisch Kranke!

Der Wegfall von drei Vierteln aller psychiatrischen Klinikbetten blieb auch für die Psychiater nicht folgenlos. Ihre professionelle Situation änderte sich, als sie aus den Kliniken heraus auf dem freien Markt ihr Auskommen suchen mussten. Dies funktionierte vor dem Hintergrund des US-Krankenversorgungssystems (Dutzende von Millionen Menschen mit wenig oder gar keinem Einkommen sind nicht krankenversichert) nur deswegen, weil die Psychiater ihre Zeit den (privat-) versicherten Menschen mit leichteren psychischen Störungen verkauften - stundenweise, mit Sätzen von um die 140 US Dollar die Stunde.

Die vermeintliche Unfähigkeit der Psychiater zum Diagnostizieren und sei es auch nur des einfachsten Unterschieds zwischen „krank“ und „nicht krank“ - hatte auch einen großen Einfluss auf das Fach Psychiatrie in dem Sinne, dass man sich um eine begriffliche Klärung von Diagnosen bemühte und explizite Kriterien für alle psychiatrischen Diagnosen formulierte. Zwar wurde [44] und wird noch immer [42] die Kritik geäußert, dass man dabei die Validität der psychiatrischen Diagnosen deren Reliabilität geopfert habe, aber ohne Reliabilität geht es eben auch wirklich nicht.

Die Auswirkungen der Publikation von Rosenhan waren also erheblich. War das alles nun insgesamt gut oder schlecht? - Frau Cahalan lässt diese Frage offen, insbesondere auch die Frage, ob Rosenhans Tun im Nachhinein als verwerfliche Täuschung anzusehen ist oder als zwar gelogene, aber dennoch sehr wirksame „heilsame“ Kritik, von Zuständen, die dringend geändert gehörten. „Letztendlich glaube ich, dass er auf etwas Reales aufmerksam gemacht hat“ ${ }^{25}$, schreibt sie gegen Ende ihres Buchs. „Rosenhans Arbeit, so übertrieben und unehrlich es auch war, berührte die Wahrheit indem es um sie herumtanzte. “ - So kann man es in intellektuellen Kreisen natürlich ausdrücken; in der Wissenschaft jedoch wird definitiv nicht um die Wahrheit getanzt! Vielmehr wird um sie gerungen, und Pseudopatienten, die Lügen erzählen, erscheinen - verglichen mit dem in der Wissenschaft üblichen Methodenarsenal - als zur Auffindung von Wahrheit eher ungeeignet.

25 „In the end I believe that he exposed something real. Rosenhan's paper, as exaggerated, and even dishonest as it was, touched on truth as it danced around it."
Insbesondere dann, wenn zugleich durch Genauigkeit, die nicht vorhanden war, die wissenschaftliche Wahrheit vorgetäuscht wird. Die angeführten frappierend exakten Angaben zur Anzahl (11,3\%) und Zeitdauer (6,8 min.) der Kontakte der Pseudopatienten mit dem Personal sowie zur Anzahl der insgesamt verabreichten Tabletten entsprechen zwar dem Anspruch und dem Format des Wissenschaftsfachblatts Science, passen aber nicht zu den offensichtlich sehr begrenzten und wenig genauen Instruktionen an Rosenhans 2 bekannte Pseudopatienten und deren Möglichkeiten (sie verfügten nicht über eine Stoppuhr). Einmal ganz von der Tatsache abgesehen, dass es 6 von 8 Pseudopatenten aller Wahrscheinlichkeit gar nicht gab.

Patienten, die keine waren, gab es schon vor (Nelly Bly im vorletzten Jahrhundert) und auch nach Rosenhan, wobei die Nichtpatienten vor Rosenhan durchaus existierten, die nach ihm nicht. Lauren Slater [29] publizierte zwar etwas, das zunächst nach einer Replikation der Rosenhan-Studie aussah, sich später jedoch als Replikation der Rosenhan-Fake-News entpuppte: Auch diese Studie war frei erfunden und hatte erneut Robert Spitzer auf den Plan gerufen, der - im respektablen Journal of Nervous and Mental Disease (Band 193) - eine Arbeit mit dem Titel: „Eine Antwort auf eine Nicht-Antwort auf Kritik an einer Nicht-Studie“ (A Response to a Nonresponse to Criticisms of a Nonstudy) publizierte [21]. Aberwitziger geht es kaum noch!

Abschließend sei noch kurz auf die Frage eingegangen, wie es überhaupt sein konnte, dass Rosenhan seine nicht sehr inhaltsreiche Studie überhaupt in Science, dem schon damals weltweit renommiertesten Fachblatt in der Wissenschaft, publizieren konnte. Von den Herausgebern der Zeitschrift erhielt Cahalan auf Anfrage keine weiteren Informationen. Meine persönliche Vermutung ist, dass Rosenhan ungewollte Hilfe von einer Instanz hatte, die höher nicht sein könnte: dem damaligen US-Präsidenten Richard Nixon. Bereits im Sommer und Herbst 1972, also während der Begutachtung des Papers von Rosenhan durch die Gutachter bei Science, „spekulierte die Washington Post schon im Sommer und Herbst 1972 - also mitten im Präsidentschaftswahlkampf - über eine weitreichende politische Verschwörung unter Einschluss des Weißen Hauses“, wie man in der Wikipedia nachlesen kann. Die angespannte, fast möchte man sagen, die paranoide Atmosphäre, die damals in den USA herrschte, mag liberal denkende Gutachter mit dazu bewogen haben, die Arbeit durchzuwinken.

Literatur

[1] Abbott A. Double deception in the asylum? Nature 2019; 574: 622-623

[2] American Psychiatric Association (APA). Diagnostic and statistical manual of mental disorders, 3rd edition (DSM-III). Washington: American Psychiatric Association Press, Washington DC 1980

[3] Anonymus. McCord declares that Mrs Mitchell was forcibly held. The New York Times 19.2.1975 (https://www.nytimes.com/1975/02/19/ archives/mccord-declares-that-mrs-mitchell-was-forcibly-held-comment-from.html?_r=0; abgerufen am 20.1.2020)

[4] Cahalan S. Brain in Fire. New York, NY: Free Press 2012

[5] Cahalan S. Lancet Psychiatry 2017; 4(5): e9, doi: https://doi. org/10.1016/S2215-0366(17)30138-4 
[6] Cahalan S. The Great Pretender. The Undercover Mission that Changes our Understanding of Madness. Edinburgh, UK: Canongate 2019

[7] Cahalan S. Stanford Professor who changed America with just one study was also a liar. NY Post, 2.11.2019

[8] Eakin E. Her Illness Was Misdiagnosed as Madness. Now Susannah Cahalan Takes On Madness in Medicine. The New York Times, 2.11.2019. www.nytimes.com/2019/11/02/books/susannah-cahalan-great-pretender.html; abgerufen am 15.1.2020

[9] Engel GL. The need for a new medical model: a challenge for biomedicine. Science 1977; 196: 129-136

[10] Ghaemi N. NIMH: A Requiem for DSM - and its Critics. A new generation will reject DSM, and the anti-biological critics of DSM too. Psychology Today, 3.5.2013. www.psychologytoday.com/us/blog/ mood-swings/201305/nimh-requiem-dsm-and-its-critics; abgerufen am 19.1.2020

[11] Goffman E. Asylums. New York: Doubleday 1961

[12] Gottesman I, Hooley J, Lenzenweger M, et al. Brendan A. Maher (Faculty of Arts and Sciences - Memorial Minute. The Harvard Gazette, 7. 10.2010. news.harvard.edu/gazette/story/2010/10/brendan-arnold-maher/; abgerufen am 20.1.2020

[13] Gottesmann I, Lenzenweger M, Lenzenweger M. Obituary: Brendan A. Maher (1924-2009) American Psychologist 2009; www.academia. edu/17124631/Obituary_Brendan_A._Maher_1924_2009_; abgerufen am 20.1.2020

[14] Jaspers K. General Psychopathology, Transl. by J Hoenig and WM Hamilton. Manchester University Press, The University of Chicago Press 1963

[15] Jaspers K (1973) Allgemeine Psychopathologie, 7. Aufl. Springer, Berlin, Heidelberg, New York

[16] Kendell RE, Cooper JE, Gourlay AJ, et al. Dioiagnostic Criteria of American and British Psychiatrists, Archives of General Psychiatry 1971; 25: $123-130$

[17] Klerman GL, Vaillant GE, Spitzer RL, et al. A debate on DSM-III. American Journal of Psychiatry 1984; 141: 539-553

[18] Lando HA. On being sane in insane places: A supplemental report. Professional Psychology 1975; 7(1): 47-52

[19] Lane C. The NIMH Withdraws Support for DSM-5. The latest development is a humiliating blow to the APA. Psychology Today, 4.5.2013 (https://www.psychologytoday.com/ us/blog/side-effects/201305/the-nimh-withdraws-support-dsm-5; abgerufen am 19.1.2020)

[20] Lawrence JM. Brendan Maher, 84, mental health pioneer. The Boston Globe, 11.5.2009 (http://archive.boston.com/bostonglobe/obituaries/ articles/2009/05/11/brendan_maher_84_mental_health_pioneer/; abgerufen am 20.1.2020)

[21] Lilienfeld SO, Spitzer RL, Miller MB. A Response to a Nonresponse to Criticisms of a Nonstudy. The Journal of Nervous and Mental Disease 2005; 193: 745-746

[22] Maher BA. Anomalous Experience and Delusional Thinking: The Logic of Explanations. In: Oltmanns TF, Maher BA: Delusional Beliefs 1988, 15-33

[23] Maher BA. Language Disorders in Psychoses and Their Impact on Delusions. In: Spitzer M, Uehlein FA, Oepen G (Hrsg) Psychopathology and Philosophy. Berlin: Springer 1988

[24] Netflix.com. Brain on Fire, starring Chloë Grace Moretz, produced by Susannah Cahalan and Charlize Theron, released June 22, 2018
[25] Rosenhan D. On being sane in insane places. Science 1973; 179: 250-258

[26] Sarbin, TR, Mancuso, JC. Schizophrenia: Medical diagnosis or moral verdict? New York: Pergamon 1980

[27] Schneider K. Clinical Psychopathology, Transl. by WM Hamilton. LOndon: Grune \& Stratton 1959

[28] Schneider K. Klinische Psychopathologie. Stuttgart: Thieme 1980

[29] Slater L. Opening Skinner's Box: Great Psychological Experiments of the Twentieth Century. New York: WW Norton 2004

[30] Spitzer M. Symptom and Criterion in Medicine and Psychiatry. In: Weingartner P, Schurz G (eds): Logic, Philosophy of Science and Epistemology (Proceedings of the 11th international Wittgenstein-Symposium) 1987; 183-185

[31] Spitzer M. Psychiatry, Philosophy, and the Problem of Description. In: Spitzer M, Uehlein FA, Oepen G (Hrsg.) Psychopathology and Philosophy. Heidelberg: Springer 1988

[32] Spitzer M. Übersetzungen, Fehler und die Folgen. Nervenheilkunde 2003; $22: 1-3$

[33] Spitzer R, Endicott ], Robins E. Clinical criteria for psychiatric diagnosis and DSM-III. American Journal of Psychiatry 1975; 132: 1187-1192

[34] Spitzer RL. On pseudoscience in science, logic in remission, and psychiatric diagnosis: A critique of Rosenhan's „On being sane in insane places“. Journal of Abnormal Psychology 1975; 84: 442-452

[35] Spitzer RL. More on Pseudoscience in Science and the Case for Psychiatric Diagnosis. Archives of General Psychiatry 1976; 33: 459-470

[36] Spitzer RL. Values and Assumptions in the Development of DSM-III and DSM-III-R: An Insider's Perspective and a Belated Response to Sadler, Hulgus, and Agich's „On Values in Recent American Psychiatric Classification“. The Journal of Nervous and Mental Disease 2001; 189: 351-359

[37] Spitzer RL, Endicott ], Gibbon M Crossing the Border into Borderline Personality and Borderline Schizophrenia. Archives of General Psychiatry 1979; 36: 17-24

[38] Stein J. Trump Ambassador Beat and 'Kidnapped' Woman in Watergate Cover-Up: Reports; Newsweek, 11.122017 (https://www.newsweek. com/2017/12/29/donald-trump-watergate-stephen-king-marthamitchell-richard-nixon-john-744823.html; abgerufen am 20.1.2020)

[39] Widiger TA, Frances A, Warner L, Bluhm C. Diagnostic criteria for the borderline and schizotypal personality disorders. J Abnorm Psychol 1986; 95: 43-51

[40] Widiger TA, Hurt SW, Frances A, et al. Diagnostic Efficiency and DSMIII. Archives of General Psychiatry 1984, 41: 1005-1012

[41] Wikipedia 2020. Rosemary Kennedy. https://de.wikipedia.org/wiki/ Rosemary_Kennedy; abgerufen am 24.1.2020

[42] Scull A. Psychiatry and ist Discontents. Oakland: University of California Press 2019

[43] Zinkler M, von Peter S. Ohne Zwang - ein Konzept für eine ausschließlich unterstützende Psychiatrie. Recht und Psychiatrie 2019; 37: 203-209

[44] Lykken DT. What's wrong with psychology anyway? In Cichetti D, Grove W (Hg) Thinking clearly about psychology, Vol. 1: Matters of public interest. Minneapolis: University of Minnesota Press 1991 